\title{
Production de matières premières et fabrication des médicaments à base de plantes médicinales
}

\author{
Salfo OUEDRAOGO ${ }^{1 *}$, Jules YODA ${ }^{1,4}$, Tata Kadiatou TRAORE ${ }^{2}$, Mathieu NITIEMA ${ }^{1}$, \\ Bavouma C. SOMBIE ${ }^{2}$, Hermine Zime DIAWARA ${ }^{2}$, Josias B.G. YAMEOGO ${ }^{2,3}$, \\ Abdoulaye DJANDE ${ }^{4}$, Lazare BELEMNABA ${ }^{1}$, Félix B. KINI ${ }^{1}$, Sylvin OUEDRAOGO ${ }^{1}$ et \\ Rasmané SEMDE ${ }^{2}$

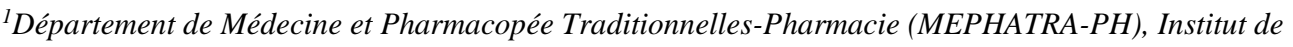 \\ Recherche en Sciences de la Santé (IRSS/CNRST), 03 BP 7047 Ouaga 03, Burkina Faso. \\ ${ }^{2}$ Laboratoire du Développement des Médicaments (LADME), Ecole Doctorale de la Santé, Université Joseph \\ Ki-Zerbo, 03 BP 7021 Ouaga 03, Burkina Faso. \\ ${ }^{3}$ Laboratoire National de Santé Publique, Ministère de la santé, 09 BP 24 Ouagadougou 09, Burkina Faso. \\ ${ }^{4}$ Laboratoire de Chimie Moléculaire et des Matériaux, Equipe de Chimie Organique et Phytochimie, \\ Université Joseph Ki-Zerbo, Burkina Faso. \\ *Auteur correspondant ; E-mail: ouedraogosalfo35@yahoo.fr ; Tel. :0022670458936
}

Received: 14-02-2021 $\quad$ Accepted: 25-04-2021 $\quad$ Published: 30-04-2021

\section{RESUME}

Les plantes constituent un réservoir pour les pharmacopées du monde. Plusieurs médicaments importants sont fabriqués à partir des substances actives d'origine végétales. En outre de nombreux médicaments modernes ont été fabriqués à partir de ces matières premières. Les plantes médicinales sont utilisées directement sous forme frâiche, sèche ou transformée, stabilisée, ou extrait ou formulée avec d'autres plantes ou excipients de synthèse. Dans tous les cas, la matière végétale utilisée pour fabriquer la forme posologique doit faire preuve de son efficacité, son innocuité et être de qualité conforme aux exigences de la Pharmacopée Européenne pour les phytomédicaments, garantissant ainsi sa sécurité d'emploi. Pour ce faire, l'Organisation Mondiale de la Santé (OMS) a mis à la disposition des états membres des guides et standards permettant d'harmoniser et de sécuriser leur utilisation. Ainsi de nombreux pays africains ont adopté ces outils après des modifications appropriées pour faire progresser la recherche et le développement $(\mathrm{R} \& \mathrm{D})$ de médicaments à base de plante. Le but de ce travail était de faire une synthèse des différentes étapes de production, de contrôle qualité et de standardisation des matières premières issues des plantes médicinales et des médicaments à base de plantes médicinales.

(C) 2021 International Formulae Group. All rights reserved.

Mots clés : Pharmacopée, médicaments, médecine traditionnelle, sécurité d'emploi, phytomédicaments.

\section{Production of raw materials and manufacturing of drugs from medicinal plants}

\begin{abstract}
Plants are a reservoir for the world's pharmacopoeias. Several important medicines are made from active substances of plant origin. In addition, many modern medicines have been made from these raw materials. Medicinal plants are used directly in fresh, dry or processed, stabilized, or extracted form or formulated with
\end{abstract}


other plants or synthetic excipients. In all cases, the herbal material used to manufacture the dosage form must demonstrate its efficacy, safety and be of a quality that meets the requirements of the European Pharmacopoeia for phytomedicines, thus ensuring its safe use. To this end, the World Health Organization (WHO) has made available to Member States guides and standards to harmonize and secure their use. Thus, many African countries have adopted these tools after appropriate modifications to advance research and development (R\&D) of plantbased drugs. The aim of this work was to make a synthesis of the different stages of production, quality control and standardization of raw materials from medicinal plants and herbal medicines.

(C) 2021 International Formulae Group. All rights reserved.

Keywords: Pharmacopoeia, drugs, traditional medicine, safe use, phytomedicines.

\section{INTRODUCTION}

Les plantes médicinales sont utilisées depuis l'antiquité comme médicaments pour la prise en charge des maladies humaines (Richard et al., 2004). Malgré les grandes avancées de la science et de la médecine moderne au cours de ces dernières décennies, ces plantes continuent de contribuer de façon importante à l'amélioration de l'état de santé des populations, notamment celle des pays en développement (Mukherjee, 2002; Bodeker and Ong, 2005; Mokosso et al., 2012; Lehmann, 2013).

De nos jours, l'intérêt pour ces plantes médicinales est en plein essor grâce à l'intégration de techniques modernes permettant d'une part d'évaluer la qualité, la sécurité et l'efficacité des métabolites secondaires et d'autre part; le rôle potentiel des médicaments élaborés à partir de ces métabolites dans les soins de santé. En effet, les plantes, avec leur grande variété de constituants phytochimiques, ont un potentiel important dans le traitement de plusieurs maladies humaines et animales (Agaie et al., 2007; Da et al., 2015; Dongock et al., 2018; Ouédraogo et al., 2019). Elles offrent une source prometteuse de médicaments. Les composés phytochimiques d'intérêt thérapeutiques peuvent provenir de nombreuses parties de la plante telles que l'écorce, les feuilles, les fleurs, les racines, les fruits, les graines, etc. avec des teneurs variables. Ces composés biologiquement actifs peuvent être isolés à partir de la plante par des procédés traditionnels à savoir la macération, la décoction, l'infusion, etc. Ils peuvent également être isolés par des techniques modernes physicochimiques et biologiques comme les fractionnements guidés, les isolements et purifications (Da et al., 2015; Dongock et al., 2018). Cependant, les substances d'origine naturelle ont le plus souvent des structures moléculaires d'une grande complexité. En effet, les plantes contiennent plusieurs centaines de constituants et certains d'entre eux sont présents à de très faibles concentrations. Malgré les procédures analytiques chimiques modernes disponibles, les recherches phytochimiques ne réussissent que rarement à isoler et à caractériser tous les métabolites secondaires présents dans l'extrait de plante. En dehors de cela, les constituants végétaux varient considérablement en fonction de plusieurs facteurs intrinsèques et extrinsèques tels les facteurs génétiques, culturels et environnementaux que sont le lieu de récolte, l'âge et la partie de la plante récoltée, la période et l'heure de récolte, la méthode de récolte, le séchage, le stockage, le transport de la matière première, etc. qui peuvent influencer leur qualité. En plus, certains constituants végétaux sont thermolabiles et les plantes qui les contiennent doivent être séchées, traitées à basse température. En outre, d'autres principes actifs sont détruits par des processus enzymatiques qui se poursuivent pendant la conservation.

Par conséquent, l'OMS et les différentes pharmacopées ont fournis des outils techniques (WHO, 2003a; Franz and Wang, 2015; Ph.Eur., 2019a; WHO, 2019) avec des modifications appropriées par région pour harmoniser la recherche et le développement (R\&D) de médicaments issus de la médicine traditionnelle (Kasilo et al., 2019). Ces outils ont permis d'avoir une qualité définie en termes de sécurité d'emploi et de protection de 
la santé publique. Cette qualité est assurée par le respect des procédures de contrôles présentées dans le dossier d'Autorisation de mise sur le marché (AMM) déposé en vue de la commercialisation du produit fini. Pour ce faire, le contrôle qualité et la standardisation des matières premières, des produits intermédiaires et finis permettront de garantir des produits de qualité pharmaceutique constante et reproductible (Yadav and Dixit, 2008). L'ensemble de ces outils et techniques vise à répondre au besoin d'intégration de la médicine traditionnelle ainsi que les produits qui en sont issus dans le système de santé surtout des pays africains. Pour y parvenir de nombreux laboratoires consacrent des ressources importantes pour l'investigation des médicaments issus de la médicine traditionnelle. L'objectif de cette étude était de faire la synthèse des méthodes utilisées dans le processus de production de matières premières et de préparations issus des plantes médicinales.

\section{PLANTES MEDICINALES}

Selon la $\mathrm{X}^{\text {ème }}$ édition de la Pharmacopée française (Ph.Fr., 2012), les plantes médicinales "sont des drogues végétales au sens de la Pharmacopée européenne dont au moins une partie possède des propriétés médicamenteuses". Ces plantes médicinales peuvent également avoir des usages alimentaires ou hygiéniques. En d'autres termes, les plantes médicinales sont des plantes utilisées en médecine traditionnelle et/ou moderne dont au moins une partie renferme une ou des substances qui possèdent des propriétés thérapeutiques (WHO, 2002b).

Leur action provient des composés phytochimiques (métabolites primaires ou secondaires) qui agissent seul ou de la synergie entre ces différentes composées. Le matériel végétal destiné à être utilisé en thérapeutique constitue la drogue végétale. Il peut s'agir de la plante entière, d'une ou de plusieurs parties de plantes ou d'extraits (Lehmann, 2013; Limonier, 2018). Ces drogues végétales proviennent de plantes sauvages ou cultivées. En fonction de la provenance de la drogue, la teneur en principes actifs peut varier de manière plus ou moins importante, entraînant une activité à priori variable d'un lot à un autre. Il faut donc faire attention à l'origine géographique et aux conditions écologiques (altitude, degré de fertilisation du sol, caractère sauvage ou cultivé de la plante). La qualité finale de la drogue dépend des conditions de culture, de récolte, de séchage, de fragmentation et de stockage. Les drogues doivent être exemptes d'éléments étrangers tels que la poussière, souillures, signes d'infection fongique ou de contamination animale. Si une stérilisation s'avère indispensable, il faut prouver qu'elle n'altère pas les constituants de la plante et qu'elle ne subsiste aucun composé nocif à l'issu du traitement (WHO, 2000b; OMS, 2013).

\section{Origine des plantes médicinales}

Les plantes médicinales sont caractérisées par deux origines. Ce sont les plantes spontanées dites "sauvages" ou "de cueillette", et les plantes cultivées (Chabrier, 2010).

\section{Plantes sauvages}

Cette catégorie constitue les plus anciennement utilisées et représentant encore aujourd'hui un pourcentage notable du marché mondial. Leur répartition et développement dépend de plusieurs facteurs tels que le type de sol et surtout du climat (Chabrier, 2010). Ces plantes sont en effet influencées par la température, la latitude, l'altitude, la composition du sol, etc. Ces conditions édaphiques font de ces plantes des véritables réservoirs de spécificités génétiques.

\section{Plantes cultivées}

Ces plantes permettent, grâce aux techniques de culture standardisées d'obtenir des matières premières de bonne qualité en quantité suffisante et homogènes. En effet, la culture des plantes médicinales répond à des directives de l'OMS sur les bonnes pratiques agricoles et des bonnes pratiques de récolte (BPAR) relatives aux plantes médicinales (WHO, 2003a). Elles s'appliquent à la culture, à la récolte des plantes médicinales et à certaines opérations postérieures à la récolte. Les directives peuvent être adaptées à la réglementation en vigueur dans les différents 
pays. En plus de tous ces bénéfices sur la qualité, la culture pallie la dispersion ou la disparité des peuplements naturels. L'importante diversité créée au sein des espèces cultivées, bien que très inférieure à celle de la flore spontanée, constitue aussi un réservoir de spécificités génétiques (Chabrier, 2010).

\section{Critères de sélection des plantes médicinales}

Un des critères de choix les plus importants pour la sélection est la preuve de la sécurité d'emploi à travers des preuves d'innocuité et d'efficacité. En effet, à partir des enquêtes ethnobotaniques, des informations sur l'utilisation des plantes médicinales par des tradipraticiens et les populations sont rassemblées (Chegaing et al., 2020). Sur la base de ces informations, des études ethnopharmacologiques in vitro, ex vivo et in vivo (chimie, pharmacologie, toxicologie, microbiologie, etc.) sont entreprises afin de mieux étudier la matière végétale dans sa composition, ainsi que ses potentialités thérapeutiques. Au cours de ces études, les informations sur la disponibilité des individus de l'espèce des plantes médicinales ainsi que des méthodes de cultures, de récolte et de protection sont rassemblées. Le choix des sites de cultures et récoltes est réalisé à travers des études de standardisation. La variabilité des constituants des drogues ou des préparations à base de plantes en raison de facteurs intrinsèques et extrinsèques sont prise en compte.

\section{Matières premières issues des plantes médicinales}

Ce sont des substances issues de plante entière ou partie de plantes (principes actifs, excipients, solvants, gaz...) utilisées sèches, stabilisées ou desséchées pour la fabrication du médicament. Leur qualité est définie soit par une monographie soit par une pharmacopée (Limonier, 2018). Ces matières végétales comprennent, outre les plantes entières, les parties de plantes telles que les sucs, gommes, huiles grasses, huiles essentielles, résines et poudres sèches issus de feuilles, fleurs, fruits, graines, tronc, bois, écorce, racines, rhizome et autres parties, entières, fragmentées ou en poudre. Ces matières sont obtenues selon divers procédés traditionnels ou modernes (WHO, 2000b; Chabrier, 2010). Les matières premières entrant dans la production doivent répondre à un cahier des charges complet et rigoureux. Celui-ci correspond aux impératifs règlementaire et éthique du médicament à base de plante, qui sont d'autant plus exigeants lorsque les molécules sont d'origine naturelle, et non de synthèse. Tout d'abord, une sélection des plantes identifiées botaniquement est primordiale. Un mode de culture biologique ou de l'agriculture raisonnée est préférable. Ensuite, le recours à un procédé d'extraction complet est souhaité. En effet, il garantit le respect de l'intégralité et de l'intégrité des principes actifs contenus dans la plante d'origine de façon à restituer sa composition moléculaire. De plus, de nombreux contrôles sont réalisés selon les exigences de la règlementation en vigueur (Wichtl et al., 2003).

\section{Composition chimique des plantes}

La plante possède une composition chimique complexe, constituée de plusieurs substances. Cette composition complexe résulte de l'interaction de la plante avec son environnement. En effet, elle puise par ses racines dans le sol l'eau, les minéraux (oligoéléments et macroéléments) nécessaires à sa croissance. Elle réalise la photosynthèse dans ses feuilles, elle élabore des molécules complexes appelées composés organiques (Chabrier, 2010).

Les substances élaborées par les plantes sont classées en deux groupes :

- Métabolites primaires qui sont nécessaires à la vie végétale et qui ne présentent qu'une activité pharmacologique de base (les glucides tels que la cellulose et l'amidon, les lipides, les enzymes...);

- Métabolites secondaires ou spécialisés qui sont de composition plus complexe et généralement regrouper dans les grandes familles chimiques telles que les polyphénols, les terpénoïdes et les alcaloïdes (Fatiha, 2019).

$\mathrm{Ce}$ dernier groupe de métabolites renferme les molécules les plus utilisées en 
thérapeutique. Elles sont également d'un grand intérêt pour la plante car elles la protègent des rayons du soleil, des oxydations et interviennent comme signaux d'échange avec son environnement (pour se protéger d'autres espèces ou pour attirer les insectes pollinisateurs).

Malgré de nombreuses recherches sur les drogues végétales, très peu de métabolites secondaires ont pu être isolé et identifié (Chabrier, 2010; Fatiha, 2019).

\section{Méthodes de préparation et formes de présentation médicamenteuse \\ Méthode de préparation}

Les méthodes de préparation des produits à base de plantes utilisent des procédés préalables de la drogue (concassage, broyage, pressage, exsudation ou extraction par différents techniques) selon la forme recherchée avec des solvants appropriés (l'eau, l'éthanol, les mélanges hydroéthanoliques, la glycérine, l'huile, l'acétone, etc.). Le mode de la préparation doit être maitrisé et validé car elle influence la composition et la teneur en substances actives recherchés. En cas d'utilisation de solvant de classe II et III, leur teneur résiduel doit être établi.

Les préparations peuvent être sous forme de poudres, de tisanes, d'extraits fluides/ mous/secs, de teintures mères, de macérâtes glycérinés, de suspensions intégrales de plantes fraiches (SIPF) et des extraits fluides de plantes standardisées (EPS). Le mode de préparation conditionne la forme, la composition et la teneur en substance active recherchée.

Il n'y a pas de solvant type pour une extraction, mais la nature de ce dernier conditionne les caractéristiques de l'extrait. La méthode d'extraction doit donc être choisie avec minutie afin de retenir les constituants recherchés. L'utilisation de l'eau, et plus tard de l'alcool (éthanol) comme solvants est généralement adaptée pour la préparation des phytomédicaments étant donné la corrosivité (Chabrier, 2010) et la toxicité de certains solvants.

\section{* Différents types d'extraits}

Les extraits sont des préparations liquides (extraits fluides et teintures), de consistance semi-solide (extraits mous ou fermes) ou solide (extraits secs), obtenues à partir de drogues végétales. Ces extraits peuvent être séchés par différentes techniques telles que l'évaporation par la chaleur, la lyophilisation ou l'atomisation, etc. (Limonier, 2018). Ces extraits peuvent être titrés, standardisés, quantifiés (ou normalisés) et purifiés. Les extraits titrés sont ajustés au moyen d'une substance inerte ou en mélangeant des lots d'extraits, avec une tolérance acceptable à une teneur donnée en constituants ayant une activité thérapeutique connue. Les extraits quantifiés sont ajustés à une fourchette définie de constituants en mélangeant des lots d'extraits. Les autres extraits sont définis par leur procédé de production (état de la drogue végétale, solvant, conditions d'extraction) et leurs spécifications (Ph.Eur., 2008c). Le large spectre d'activité des plantes médicinales est dû à leur complexité chimique, c'est pourquoi elles possèdent souvent plusieurs indications thérapeutiques (Société.Française, 2007).

\section{* Procédés d'extraction les plus rencontrés}

1) La macération : procédé de dissolution et d'extraction partielle par un solvant donné, consistant à maintenir, pendant plusieurs heures, la matière première en contact, à froid, avec ce solvant. Le mélange (matière première et solvant) doit être remué à intervalle régulier. Le produit obtenu est un macéré ou un macérât.

2) La lixiviation ou percolation : procédé de dissolution et d'extraction partielle consistant à épuiser une matière première en faisant filtrer, à travers elle, un solvant froid ou chaud qui entraîne, avec lui, tout composé soluble. Le produit obtenu est un lixiviat. Par exemple le café filtré est obtenu par lixiviation.

3) L'infusion : opération de dissolution extractive consistant à verser sur la matière première de l'eau bouillante, à maintenir le contact pendant un certain temps puis à laisser refroidir. Le produit obtenu est appelé infusé.

4) La décoction : procédé de dissolution et d'extraction partielle, par exemple par l'eau ou l'alcool, consistant à maintenir la matière première en contact avec le solvant, à l'ébullition. Le produit est un décocté. 


\section{* Rendement d'extraction}

Il s'agit des poids indicatifs des constituants chimiques extractibles du produit brut sous différents solvants. Préalablement à l'extraction, les drogues, qui se présentent sous forme de fragments séchés, sont broyées jusqu'à atteindre un degré de granulométrie adapté à une dissolution optimale des constituants à isoler. La granulométrie retenue représente un compromis entre degré d'extraction maximale, filtration aisée et absence de tassement. Dans le cas d'une extraction en milieu aqueux, la drogue est humidifiée pour empêcher tout gonflement tardif. Après l'extraction quel que soit le solvant utilisé, le rendement de l'extraction est déterminé à partir de la formule suivante :

\section{$\mathbf{R}(\%)=\mathbf{P} \times 100 / \mathbf{P}_{0}-\mathbf{T H R}$}

$\mathrm{R}$ : Rendement d'extraction

THR : Taux d'humidité résiduelle

$P$ : Poids (g) d'extraits secs obtenus après lyophilisation

$\mathrm{P}_{0}$ : Poids ( $\mathrm{g}$ ) de la prise d'essai de matière végétale

\section{Formes de présentation médicamenteuses}

Les matières premières à base de plantes médicinales sont utilisées soit sous leur forme «native » ou brute, soit sous des formes galéniques plus élaborées comme les teintures, extraits, tisanes, opiats, électuaires (préparation pharmaceutique de consistance molle, formée de poudres mélangées à du sirop, du miel, des pulpes végétales), thériaques (électuaire contenant de nombreux principes actifs dont l'opium), loochs (médicament sirupeux, adoucissant, composé essentiellement d'une émulsion et d'un mucilage) et trochisques (médicament composé de substances sèches pulvérisées et moulées en forme de cône, destiné aux fumigations par combustion) (Chabrier, 2010).

Selon la définition de l'OMS (WHO, 2000b, 2002b; OMS, 2013), les médicaments à base de plantes contiennent comme ingrédients actifs des parties de plantes ou des matières végétales à l'état brut ou transformé. Ils se présentent généralement sous forme de préparations liquides, solides (extrait en poudre) ou semi-solides (visqueuses). Ils sont préparés par macération, percolation ou distillation (huiles volatiles). Les extraits solides ou en poudre sont préparés par évaporation des solvants utilisés dans le processus d'extraction de la matière première. Par rapport aux drogues synthétiques bien définies, les extraits issus de plantes médicinales présentent des principes actifs souvent inconnus avec une standardisation, une stabilité et un contrôle de la qualité pas faciles à réaliser.

L'introduction de la technologie moderne dans la production commerciale de produits à base de plantes a entrainé un changement de paradigme des formes traditionnelles de préparations vers des formes pharmaceutiques modernes plus efficaces et aux effets secondaires réduits (Council, 2009). Les médicaments à base de plantes médicinales peuvent être sous différentes formes galéniques à savoir solide (comprimé, capsule...), semisolide (pommade, crème...), liquide (solution, sirop, suspension, ...). Ces présentations peuvent être fabriquées sous forme de libération conventionnelle ou modifiée. Ces formes galéniques sont à la fois un système de présentation, de conservation des médicaments à base de plantes et un système de mise à disposition de leur substance active à l'organisme du patient. C'est un élément essentiel de l'acceptabilité pour le patient (observance), de l'efficacité, du bon usage et de la sécurité d'emploi du médicament (ajustement possible de la dose) par rapport aux formes traditionnelles. De nouvelles méthodes telles que les techniques d'encapsulation permettent d'obtenir des formes encore plus nouvelles.

Elles utilisent en plus des substances actives des excipients (matières inactives) pour constituer la forme du médicament tel qu'il sera utilisé chez un patient. Les différentes formes galéniques les plus courantes sont résumées dans le Tableau 1 (Le Hir, 2009).

\section{CONTROLE QUALITE ET STANDARDISATION}

Le contrôle de qualité a pour but de vérifier les normes qualitative et quantitative préalablement établies sur la matière première, les produits intermédiaires et le produit fini. La 
standardisation consiste à uniformiser les procédures de qualité à toutes les étapes de fabrication, depuis la drogue de départ en passant par les extraits jusqu'aux produits finis, afin d'aboutir à des produits de qualité reproductible. En général, tous les médicaments, qu'ils soient synthétiques ou d'origine végétale, doivent satisfaire aux exigences fondamentales d'innocuité et d'efficacité (WHO, 2000a, 2002a; EMA, 2005).

Pour les produits à base de plantes médicinales, la standardisation doit englober l'ensemble des procédés optimisés, de la culture de la plante médicinale à son application clinique. En effet, selon l'OMS (WHO, 2000a), la standardisation et le contrôle de la qualité des plantes médicinales sont le processus d'évaluation physico-chimique des médicaments bruts couvrant des aspects tels que la sélection et la manipulation des matières premières, la sécurité, l'efficacité et la stabilité $\mathrm{du}$ produit fini. Elle comprend un contrôle botanique (examen des caractères organoleptiques, examen macroscopique et microscopique), un contrôle chimique (substances étrangères, teneur en cendres totales, teneur en humidité résiduelle, rendement extractives, ...) et de contaminants ou d'impuretés (résidus de pesticides, éléments traces métalliques, mycotoxines, contamination microbienne, radioactivité, méthanol et isopropanol) (Lehmann, 2013).

\section{Contrôle botanique}

L'identification botanique permet de donner le nom du genre, de l'espèce et de l'auteur qui a décrit la plante. Une brève description de la partie de la plante utilisée dans la fabrication du médicament (feuille, fleur, racine, ...) devrait être donnée en précisant si la plante est utilisée à l'état frais, à l'état desséché ou après traitement traditionnel. Des échantillons types, représentatifs de chaque lot de la préparation devront être authentifiés par un botaniste qualifié et un herbier devrait être constitué et conservé.

\section{Examen des caractères organoleptiques}

L'examen des caractéristiques organoleptiques englobe les analyses sur la forme générale, la couleur, l'odeur, la saveur et la texture de la drogue. Il permet parfois de reconnaître immédiatement la drogue végétale, de vérifier son degré de pureté selon la présente ou l'absence d'éléments étrangers, de moisissures, etc. et éventuellement de détecter une adultération ou une falsification (Isérin et al., 2001; Lehmann, 2013; Ph.Eur., 2016). L'observation de la forme générale permet une identification rapide de certaines drogues. La couleur peut permettre de différencier des drogues voisines. En plus, il arrive souvent qu'un changement de couleur signale une adultération par de mauvaises conditions de séchage ou de conservation. Certaines familles botaniques se reconnaissent à l'odeur caractéristique de certains types de composants qu'ils renferment. D'autres familles botaniques se reconnaissent aisément à leur saveur (âcre, brûlante, amère, astringente) justifiant ainsi leur emploi comme apéritifs et stomachiques. La texture de certaines drogues (étroitement liée à la présence de certains types de poils) constitue également un élément caractéristique de plusieurs familles botaniques.

\section{Examen macroscopique et microscopique} Examen macroscopique

L'examen macroscopique sur la drogue entière ou incisée permet de différencier des drogues voisines. Il s'agit d'observer la présence ou l'absence de caractéristiques macroscopiques telles que la nervation, les stomates. De la même façon, une observation minutieuse permet de distinguer les fruits (présence ou absence de pédoncule au niveau des diakènes). A partir de l'écorce, il est observé par exemple de cassure fibreuse contrairement à celle d'autres espèces dont la cassure est irrégulière (Ph.Eur., 2019b).

\section{Examen microscopique}

Le contrôle microscopique sur la drogue en coupe ou pulvérisée permet l'identification de la bonne variété et la recherche des adultérants. L'étude microscopique peut permettre de repérer certains éléments propres à certaines familles botaniques tels les poils secrétaires octacellulaires, les poils tecteurs, des concrétions d'oxalate de calcium, la forme des grains d'amidon, la forme de l'épiderme à 
parois cellulaire, le type de stomate, la forme du Pappus et de la trachéide. L'examen microscopique permet de détecter d'éventuelles falsifications ou substitutions de drogues végétales par d'autres drogues toxiques ou également par de drogue de moindre qualité pharmaceutique (DPSNSO, 2015; Ph.Eur., 2019b).

\section{Les méthodes d'identification chimique}

L'identification d'une drogue végétale repose généralement sur la mise en évidence de certains constituants issus du métabolisme secondaire. En ce qui concerne les contrôles de qualité à effectuer, ils sont mentionnés dans les monographies des Pharmacopées (dosage des principes actifs, teneur en eau, cendres totales, présence d'éléments étrangers, recherche de pesticides, métaux lourds, radioactivité, etc.). Pour identifier les espèces végétales sans ambiguiité, les fabricants doivent appliquer les BPAR relatives à l'identification des plantes. Les producteurs et les cueilleurs de végétaux doivent respecter plusieurs principes quand ils procèdent à l'identification et à l'authentification des espèces végétales dans divers documents détaillés. Pour obtenir des précisions à ce sujet, il convient de consulter les documents intitulés (WHO, 2000b, 2003a; Ph.Eur., 2011).

\section{Analyse chimique qualitative}

Il s'agit de l'identification et de la caractérisation de la drogue en ce qui a trait aux constituants phytochimiques (Tarpaga et al., 2020). Il utilise différentes techniques analytiques pour détecter les constituants actifs. Les techniques de criblage phytochimique impliquent souvent des extractions avec des solvants appropriés (Tableau 2), la purification et la caractérisation des principes actifs d'importance pharmaceutique. Elles passent nécessairement par des tests complémentaires (les tests en tubes et les tests sur plaques chromatographiques...). Les tests en tube sont des tests de criblages préliminaires et peuvent être confirmés ou infirmés par les criblages utilisant la chromatographie sur couche mince (CCM) (Ouédraogo et al., 2019). Les tests de criblage préliminaires en tube sont des réactions d'identifications permettant de caractériser certains constituants ou familles de constituant à l'aide de réactions chimiques simples aboutissant en général à une coloration ou à une précipitation (Evans, 2002; Kokate et al., 2004; Bekro et al., 2007; Khandelwal, 2008; Siddiqui et al., 2009). Grâce à différents protocoles standards décrits dans la littérature, il est possible de caractériser les huiles essentielles et matière grasse, les stérols et triterpènes, les caroténoïdes, les coumarines, les alcaloïdes, tanins et autres polyphénols, les anthraquinones, les saponosides, les polyuronides (pectine, les mucilages, gommes) et les composés réducteurs. Les criblages phytochimiques sur les chromatoplaques s'effectuent suivant des méthodes décrites dans la littérature (Chaaib Kouri, 2004 ; Bekro et al., 2007; Mamyrbékova-Békro et al., 2008). Il s'agit de chercher ces mêmes groupes chimiques par la CCM. En pulvérisant ces réactifs spécifiques sur le chromatogramme développé, il est observé les spots colorés indiquant la présence des composés ou groupes chimiques recherchés.

Dosage de groupes chimiques d'intérêt, substances actives ou marqueurs

L'analyse chimique quantitative permet d'estimer la quantité des principaux composés. Dans le processus de standardisation, les dosages sont nécessaires à cause des variations quantitatives saisonnières ou journalières en principes actifs ou traceurs. De plus, selon la directive 2001/83/EC, un dosage est indispensable si la drogue renferme un constituant toxique. La pharmacopée tolère une certaine souplesse dans les normes qu'elle impose pour chaque drogue végétale, afin de prendre en compte les variations biologiques normales dues à l'environnement, auxquelles toutes les plantes sont inévitablement soumises (Kabré et al., 2020). Par conséquent, les limites sont exprimées soit sous formes d'un maximum dans le cas de constituants toxiques (Miningou et al., 2020). Pour certaines drogues ainsi que pour les préparations galéniques titrées, une fourchette (minimum-maximum) est imposée pour les drogues à constituants actifs bien défini. Un dosage du principe actif peut être réalisé soit par chromatographie en 
phase gazeuse (CPG), s'il s'agit d'une substance volatile, soit par la chromatographie liquide à haute performance (HPLC), s'il s'agit d'une substance fixe, ou par spectrophotométrie d'absorption dans l'ultraviolet. Pour les drogues présentant un groupe de constituants actifs comme les polyphénols par exemple, souvent constitués de substance issues de chaines métaboliques caractéristiques de l'espèce botanique considérée et ne présentant que de faibles différences chimiques, il convient de procéder à un dosage global de ces constituants. En effet, les activités pharmacologiques de ces molécules appartenant à un même ensemble sont souvent très voisines sinon équivalentes. Une évaluation spectrophotométrique est habituellement pratiquée dans ce cas et le résultat du dosage global est exprimé en molécules majoritaires. Enfin, pour la drogue présentant un totum actif sans qu'aucun constituant ou groupe de constituant ne se soit avéré isolement actif, il convient de choisir avec soin un traceur analytique qui constitue l'empreinte digitale chimique de la drogue. Il est souvent nécessaire de mettre au point ou de développer une méthode de dosage du traceur. Examen chromatographique

Les empreintes chromatographiques peuvent être utilisées dans le contrôle de la qualité des matières végétales médicinales. Les analyses chromatographiques comprennent principalement la chromatographie sur couche mince (CCM), la CPG et la chromatographie phase liquide à haute performance (CLHP). D'autres techniques telles la CCM quantitative (CCMQ) et la CCM haute performance (CCMHT) peuvent déterminer l'homogénéité d'un extrait végétal. La Chromatographie en couche surpression (OPLC), spectrométrie infrarouge et UV-Visible, spectrométrie de masse (MS), CPG, chromatographie liquide (LC) utilisée seule ou en combinaison comme CPG-MS et LC-MS, et résonance magnétique nucléaire (RMN), les techniques électrophorétiques, notamment les techniques de chromatographie par césure, sont des outils puissants, souvent utilisés pour standardiser et contrôler la qualité tant du matériau brut que du produit fini. Les résultats de ces techniques sophistiquées fournissent une empreinte chimique quant à la nature des produits chimiques ou des impuretés présentes dans la plante ou l'extrait (Mamyrbékova-Békro et al., 2008).

\section{Teneur en humidité}

Selon la Pharmacopée européenne, la perte à la dessiccation est la perte de masse exprimée en pourcentage $\mathrm{m} / \mathrm{m}$. Le mode opératoire est précisé dans chaque monographie de plante. La dessiccation peut s'effectuer jusqu'à masse constante ou pendant une durée déterminée, soit dans un dessiccateur en présence de pentoxyde de diphosphore, soit sous vide avec indication d'un intervalle de température, à l'étuve ou sous vide poussé. Il est à noter qu'un pourcentage d'eau trop élevé permet à un certain nombre de réactions enzymatiques de se développer, entraînant des conséquences néfastes sur l'aspect des drogues, leurs caractères organoleptiques, leurs propriétés thérapeutiques. En outre, une humidité résiduelle favorise le développement de microorganismes (bactéries, levures, moisissures) (Ph.Eur., 2008c).

La vérification du taux d'humidité permet de réduire les erreurs dans l'estimation du poids réel du matériel médicamenteux. Une faible humidité suggère une meilleure stabilité contre la dégradation du produit. La teneur admise dans une drogue pour une bonne conservation ne doit guère excéder $10 \%$. Une fois bien déshydratée (THR $\leq 10 \%$ ), la drogue végétale est conservée dans des récipients étanches à l'air et à l'eau sous forme entière, fragment ou poudre (Ph.Eur., 2008c).

\section{Contaminants organiques étrangers}

Les médicaments à base de plantes médicinales doivent être fabriqués à partir de la partie indiquée de la plante et doivent être exempts d'autres parties de la même plante ou d'autres plantes. Les produits à base de plantes médicinales devraient être entièrement exempts de moisissures ou d'insectes, y compris d'excréments et de contaminants visibles comme le sable et les pierres, de matières étrangères toxiques et nocives et de résidus chimiques. Les matières animales telles que les insectes et les contaminants microbiens " invisibles ", qui peuvent produire des toxines, 
font également partie des contaminants potentiels des drogues (EMA, 2002; WHO, 2003b, 2004). L'examen macroscopique peut facilement être utilisé pour déterminer la présence de corps étrangers, bien que la microscopie soit indispensable dans certains cas particuliers (par exemple, l'amidon ajouté délibérément pour "diluer" le matériel végétal). De plus, lorsque des matières étrangères consistent, par exemple, en un résidu chimique, la CCM est souvent nécessaire pour détecter les contaminants (AOAC, 2005).

\section{Teneur en cendres}

Le taux de cendre permet d'évaluer le degré de propreté de la plante. L'expression cendres totales est un terme se rapportant à la partie inorganique d'un échantillon alimentaire restant après que l'échantillon a brûlé à $600^{\circ} \mathrm{C}$ pendant deux heures. Ce résidu contient les oligo-éléments essentiels tels que le calcium, le phosphore, le sodium, le potassium, le magnésium et le manganèse. Il s'agit de critères permettant de juger de lidentité et de la pureté de la drogue. Les valeurs souvent rechercher sont: le taux en cendres totales, cendres sulfatées, cendres solubles dans l'eau, cendres insolubles dans l'acide, etc. (AOAC, 2005). Dans les plantes, le contenu minéral varie de moins de $1 \%$ à plus de $12 \%$.

\section{Résidus de produits phytosanitaires et} pesticides

La culture des plantes médicinales se substitue à la récolte manuelle de plantes sauvages, entraînant une généralisation de l'utilisation de produits phytosanitaires (pesticides, insecticides, herbicides, fongicides...). Toute substance ou association de substances destinée à repousser, détruire ou combattre les ravageurs et les espèces indésirables de plantes et d'animaux, causant des dommages ou se montrant autrement nuisibles durant la production, la transformation, le stockage ou la mise sur le marché de substances médicinales d'origine végétale est considérée comme pesticide. La Pharmacopée européenne donne des valeurs limites de tolérance (en $\mathrm{mg} / \mathrm{kg}$ ) pour 34 pesticides (Tableau 3) (Sofowora, 2010). Les drogues à base de plantes médicinales sont susceptibles de contenir des résidus de pesticides qui s'accumulent lors des pratiques agricoles, comme la pulvérisation, le traitement des sols pendant la culture et l'administration de fumigants pendant le stockage.

\section{Élément traces-métalliques}

La contamination des plantes médicinales par des métaux toxiques comme le mercure, le plomb, le cuivre, le cadmium, l'arsenic par exemple, peut être accidentelle ou intentionnelle. Elle peut être attribuée à de nombreuses causes, y compris la pollution de l'environnement, et peut poser des dangers cliniquement pertinents pour la santé de l'utilisateur et devrait donc être limitée (AOAC, 2005). Une détermination simple et directe des métaux lourds peut être trouvée dans de nombreuses pharmacopées et est basée sur des réactions colorées avec des réactifs spéciaux tels que le thioacétamide ou le diéthyldithiocarbamate, et la quantité présente est estimée par comparaison avec une norme. Les recommandations en matière de contamination par les métaux lourds ou métaux toxiques prévoient des limites qui sont fonction des plantes. Les concentrations limites dans le matériel végétal brut des métaux lourds fournis par l'OMS sur la base d'une revue des règlementations en vigueur dans différents pays (WHO, 2007) sont respectivement le Cadmium 1,0 ppm, le Plomb 10,0 ppm, le

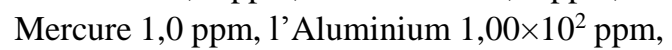
le Chrome $1,00 \times 10^{2} \mathrm{ppm}$, l'Etain 10,0 ppm, le Manganèse 10,0 ppm, le Molybdène 10,0 ppm,... Il est par ailleurs exigé que la recherche des métaux lourds soit réalisée avec le spectromètre d'absorption atomique sauf exception justifiée et autorisée. Selon la nature et l'origine de la drogue, d'autres métaux lourds peuvent être recherchés : Cuivre

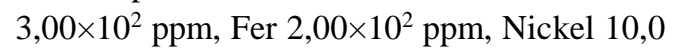
ppm, Zinc $1,00 \times 10^{2}$, etc. (Ph.Eur., 2008a; Lehmann, 2013).

\section{Recherche de mycotoxines}

Les mycotoxines forment un groupe diversifié de composés toxiques, produits par certaines espèces de moisissures qui peuvent contaminer des produits agricoles et des denrées alimentaires. Plus de 300 mycotoxines différentes ont été identifiées, seules certaines souches peuvent produire des toxines. $\mathrm{La}$ 
production des mycotoxines a lieu lors du stockage et du transport de la drogue. Les aflatoxines sont des métabolites secondaires produits par des champignons microscopiques proliférant sur des graines conservées en atmosphère chaude et humide. Parmi les plus courants se trouvent les aflatoxines B1, B2, M1, G1 et G2 (WHO, 2000a). L'aflatoxine B1 est la plus courante et la plus toxique. Comme les aflatoxines sont très solubles dans des solvants organiques polaires comme le méthanol, il semble probable qu'elle passe facilement dans les extraits éthanoliques. Selon les directives de la pharmacopée européenne, la teneur en aflatoxines est fixée, sur la base des limites autorisées dans l'industrie agroalimentaire. Il faut noter que pour l'aflatoxine B1 une limite maximale est fixée à $2 \mu \mathrm{g} / \mathrm{kg}$ et une limite de $4 \mu \mathrm{g} / \mathrm{kg}$ pour la somme des aflatoxines B2, M1, G1 et G2 (WHO, 2000a; Lehmann, 2013). Les ochratoxines, avec comme limite de $20 \mu \mathrm{g} / \mathrm{kg}$ (FAO, 2018), sont également des métabolites secondaires d'origine fongique mais leur recherche n'est pas pertinente pour la plupart des plantes médicinales (Lehmann, 2013).

\section{Contaminants microbiens}

Les plantes médicinales peuvent être associées à une grande variété de contaminants microbiens, représentés par des bactéries, des champignons et des virus. Inévitablement, ce contexte microbiologique dépend de plusieurs facteurs environnementaux et a un impact important sur la qualité globale des produits et préparations à base de plantes. L'évaluation des risques liés à la charge microbienne des plantes médicinales est donc devenue un sujet important dans la mise en place de systèmes modernes d'analyse des risques et de maîtrise des points critiques.

Les médicaments à base de plantes médicinales contiennent normalement un certain nombre de bactéries et de moisissures, qui proviennent souvent du sol. Des mauvaises méthodes de récolte, de nettoyage, de séchage, de manipulation et d'entreposage peuvent également entraîner une contamination supplémentaire. Les procédures de laboratoire concernant les contaminations microbiennes sont définies dans les pharmacopées bien connues, ainsi que dans les directives de l'OMS (WHO, 2000a). Les valeurs limites peuvent également être trouvées dans les exigences tirées des Pharmacopées mentionnées ou autres méthodes internationalement reconnues (Ph.Eur., 2008c). En exemple, les limites de tolérances de quelques organismes vivants sont mentionnées dans le Tableau 4 (DPSNSO, 2015). Les opérations susceptibles de limiter la présence des germes par stérilisation dans les drogues sont malheureusement également capables de dégrader les constituants présents. Contamination radioactive

Une contamination dangereuse peut cependant être la conséquence d'un accident nucléaire. L'OMS, en étroite coopération avec plusieurs autres organisations internationales, a élaboré des lignes directrices en cas de contamination généralisée par des radionucléides résultant d'accidents nucléaires majeurs. Certaines publications soulignent que le risque pour la santé, en général, dû à la contamination radioactive par les radionucléides naturels n'est pas vraiment préoccupant, mais que ceux qui découlent d'accidents nucléaires majeurs comme l'accident nucléaire de Tchernobyl et de Fukushima peuvent être graves et dépendent du radionucléide spécifique, du niveau de contamination et de la quantité du contaminant consommé. Compte tenu de la quantité de plantes médicinales normalement consommée par un individu, il est peu probable qu'il y ait un risque pour la santé. Par conséquent, aucune limite n'est actuellement proposée pour la contamination radioactive (WHO, 2000a; AOAC, 2005).

Recherche de contamination par les solvants

Les médicaments à base de plantes sont préparés à partir d'un nombre limité de solvants parmi lesquels se trouve l'alcool (le méthanol, le n-butanol, l'éthanol, ...) plus rarement l'acétone, l'acétate d'ethyle, l'hexane et l'heptane. Les teneurs en méthanol et en isopropanol doivent généralement rester inférieures à $0.05 \%$ (500 ppm). Il est distingué trois catégories de solvants (DPSNSO, 2015) selon leurs risques potentiels (Tableau 5) (Ph.Eur., 2008b). La classe 1 (cancérigènes humains connus) regroupe les solvants ne 
devant pas être utilisés : benzène, tétrachlorure de carbone, 1,2-Dichloroéthane, 1,1Dichloroéthène et 1,1,1-Trichloroéthane. S'il est impossible d'éviter le recours à des solvants de classe 1, il est essentiel d'obtenir une confirmation de leur acceptabilité avant de les utiliser sur la matière première. Dans la classe 2 se placent ceux présentant des limites d'utilisation en raison de leur toxicité intrinsèque : chloroforme, cyclohexane, éthylène glycol, hexane, méthanol, pyridine, toluène, xylène, etc. Enfin la classe 3 est réservée aux faibles potentialités toxiques. $\mathrm{La}$ limite admissible pour des solvants de cette classe est inférieure ou égale à $50 \mathrm{mg} / \mathrm{jour}$ (ce qui correspond à $5000 \mathrm{ppm}$ ou $0,5 \%)$ : acétone, acide acétique, butanol, éthanol, éther éthylique, méthyléthylcétone, 1-propanol, 2propanol, etc.

Des essais de recherche de solvants résiduels sont donc réalisés. Les solvants résiduels utilisés dans les produits à usage pharmaceutique sont définis ici comme des produits chimiques organiques volatils, utilisés ou produits dans la fabrication de substances actives ou d'excipients, ou entrant dans la préparation de médicaments. La limitation dépend du type de produit utilisé, de la voie d'administration, des posologies et de la durée de traitement (DPSNSO, 2015).

\section{Analyse granulométrique}

L'analyse de la distribution granulométrique est réalisée par des tests de la Pharmacopée Européenne (Ph.Eur., 2008c). Le tamisage est l'une des plus anciennes méthodes de classification des poudres et granulés en fonction de leur distribution granulométrique. D'autres techniques comme les méthodes d'entraînement par l'air (tamiseurs à jet d'air et à ultrasons), la microscopie ainsi que la diffraction des rayons $\mathrm{X}$ permettent en plus de la distribution des grains de mettre en évidence la morphologie et les dimensions des grains. Ce test intervient dans le choix du procédé, des excipients pour une meilleure homogénéité.

\section{Contrôle de stabilité}

Selon la Conférence Internationale de l'Harmonisation (ICH) (ICH, 2003), la stabilité est définie comme suit : «C'est l'aptitude d'un médicament à conserver ses propriétés chimiques, physiques, microbiologiques et biopharmaceutiques dans des limites spécifiées pendant toute sa durée de validité. Cette stabilité dépend, d'une part, de facteurs environnementaux (température, humidité relative et la lumière), d'autre part, de facteurs liés aux produits comme les propriétés physiques du principe actif et des excipients, du procédé de fabrication, de la nature du système récipient-fermeture et des propriétés des matériaux de conditionnement $\gg$ (Chavass et al., 2001). Un médicament est considéré comme stable lorsque ses propriétés essentielles ne changent pas, ou changent dans des proportions tolérables jusqu'à sa date de péremption. Pour cela, la stabilité des médicaments doit être surveillée selon un programme approprié et continu permettant la détection de tout problème (par exemple tout changement du taux des impuretés, du profil de dissolution) relative à la formulation du produit dans son conditionnement final (WHO, 2007). La composition chimique des drogues peut se modifier au cours du temps, en dépit des précautions prises pour le stockage. Les plantes aromatiques perdent naturellement leur huile essentielle au fil du temps, par évaporation, et ce d'autant plus rapidement qu'elles sont finement divisées. Les préparations à base de drogue végétale ou les médicaments qui en sont issus doivent garantir la stabilité de leurs constituants au fil du temps. Les médicaments présentés sous forme de solution (teintures alcooliques, sirop...) ont des durées de stabilité inférieures à celles des extraits secs ou des poudres.

\section{Validation}

La validation est le processus qui consiste à prouver qu'une méthode d'analyse est acceptable aux fins prévues pour les méthodes pharmaceutiques. En général, les études de validation doivent comprendre des 
études sur la spécificité, la linéarité, l'exactitude, la précision, la portée, la détection et les limites quantitatives selon que la méthode analytique utilisée est qualitative ou quantitative (Pinguet, 2015).

Pour une qualité constante des produits issus des plantes médicinales, il est nécessaire d'assurer une validation scientifique des procédés utilisés et un suivi périodique de la qualité et de l'efficacité des drogues. La validation est l'expression complète d'une séquence d'activité ayant pour but de démontrer et documenter qu'un médicament peut être fabriqué de façon fiable par des procédés déterminés, avec une qualité appropriée pour leur utilisation destinée. C'est l'établissement de la preuve, en conformité avec les principes de bonnes pratiques de fabrication (Raynaud, 2011). Aucune ligne directrice spécifique expliquant comment effectuer la validation analytique des produits d'origine végétale n'a été publiée à ce jour (Bellenot et al., 2012). La validation d'une procédure analytique non décrite à la Pharmacopée suit la même réglementation que les médicaments renfermant des substances actives chimiquement définies, et doit donc satisfaire aux exigences de la norme ICH Q2 (R1) (Hubert et al., 2003). L'utilisation des guides de validation de la SFSTP est également possible.

\section{CONTROLES QUALITE DES FORMES PHARMACEUTIQUES A BASE DE PLANTES}

Les contrôles des médicaments à base de plantes comportent des contrôles chimiques ainsi que des contrôles liés à la forme galénique sous laquelle se présente le médicament (essai de dissolution, de désagrégation, uniformité de masse et de teneur...), des contrôles liés aux différents excipients utilisés, des recherches d'impuretés, notamment microbiologiques, et des études d'interactions contenant / contenu (Calixto, 2000; Ph.Eur., 2019a). Les pharmacopée Européenne, USP (Pharmacopée des Etats Unis), britannique ainsi que les indications issues des monographies européennes élaborées par le comité sur les médicaments à base de plantes (HMPC) au sein de l'Agence européenne du Médicament (EMA) définissent les normes (USP, 2007; Lehmann, 2013).

\section{Contrôles chimiques}

Des dosages en un ou plusieurs constituant(s) chimique(s) sont habituellement pratiqués sur les médicaments de phytothérapie. Dans le cas des extraits standardisés, le type de constituant dosé est fonction du caractère titré, quantifié (ou normalisé) ou purifié de l'extrait.

\section{Contrôles liés à la forme galénique}

Les médicaments de phytothérapie, tout comme les médicaments allopathiques dits « conventionnels », peuvent se présenter sous un grand nombre de formes galéniques destinées à différentes voies d'administration. Les contrôles propres à la forme galénique sous laquelle ils se présentent s'imposent donc aux médicaments à base de plantes, ainsi que les contrôles des excipients utilisés lors de la mise en forme galénique (Patel et al., 2006).

\section{Formes pharmaceutiques solides destinées à la voie orale}

Les contrôles analytiques des comprimés (enrobés ou non enrobés), gélules, capsules molles, granules, etc. comprennent différents essais : détermination du temps de désagrégation ou de la vitesse de dissolution, essai de dureté / friabilité, essai d'uniformité de dosage (teneur et masse), évaluation de la teneur en eau sans compter les études de stabilité. Dans le cas des comprimés bi- ou quadri-sécables vient s'ajouter en outre un essai de sociabilité.

Essai de désagrégation / dissolution (2.9.1) : Ph. Eur.

La désagrégation est une condition généralement nécessaire mais non suffisante pour la libération du principe actif qui ne peut être absorbé qu'une fois dissout. C'est au cours de la période de recherche et développement qu'il est défini si l'essai de dissolution est 
nécessaire en routine ou bien si l'essai de désagrégation suffit. Si l'essai de dissolution est adopté, l'essai de désagrégation est alors inutile. Selon que la présence d'une forme à libération immédiate ou d'une forme à libération modifiée ou prolongée, il convient d'effectuer une ou plusieurs mesures. Le temps de désagrégation également appelé temps de délitement se détermine sur six comprimés selon la méthode de la Pharmacopée Européenne. Il convient de noter que l'essai de désagrégation est inapproprié pour les comprimés à croquer. La vitesse de dissolution peut être déterminée quant à elle selon trois procédés différents : la méthode de l'appareil à palette, la méthode de l'appareil à panier ou encore la méthode de la cellule à flux continu (Ph.Eur., 2016).

Essai de dureté / résistance à la rupture (2.9.8) $\therefore \mathrm{Ph}$. Eur

Cet essai consiste à faire subir au comprimé une pression constante jusqu'à écrasement à l'aide d'un appareil constitué de deux mâchoires se faisant face, l'une se déplaçant vers l'autre qui est fixe. La force exercée au moment de la rupture du comprimé ainsi que la position de ce dernier par rapport à l'application de la force sont mesurées à un Newton près (Patel et al., 2006 ; Lehmann, 2013 ; Brossard et al., 2016 ; Ph.Eur., 2016).

Essai de friabilité (2.9.7) : Ph. Eur.

Les comprimés sont placés dans un appareil qui leur fait subir des frottements et des chutes durant un temps déterminé. Ils sont pesés avant et après ce traitement et la perte de masse (exprimée en pourcentage par rapport à la masse initiale) est calculée. Celle-ci doit être minime $(<1 \%)$ sinon les comprimés ne supporteront pas toutes les manipulations qu'ils devront subir jusqu'à leur utilisation (Wehrlé, 2007 ; Brossard et al., 2016 ; Ph.Eur., 2016).

\section{Essai de sociabilité}

Cet essai ne concerne que les comprimés sécables, c'est-à-dire ceux qui comportent une ou deux barres de cassure. Il s'agit de vérifier que les fractions obtenues sont de masses sensiblement égales.
Formes pharmaceutiques liquides destinées à la voie orale

Les contrôles des formes pharmaceutiques liquides destinées à la voie orale comportent une mesure du $\mathrm{pH}$, la détermination de la teneur en éthanol s'il y a lieu (dans le cas des teintures, alcoolats, élixirs, sirops contenant de l'alcool...), le dosage des conservateurs tant antioxydants qu'antimicrobiens s'il y en a, un essai de dissolution, un essai de distribution de taille des particules (dans le cas des suspensions, solutions, ...), un essai de redispersabilité, une étude des propriétés rhéologiques, une mesure de la viscosité ainsi qu'une détermination de la teneur en eau (directe par exemple selon la méthode de titration de Karl FISCHER, ou indirecte par l'essai de la perte à la dessiccation).

Formes pharmaceutiques stériles (injectables, collyres, ...)

Les essais des formes pharmaceutiques stériles (ampoules injectables de Viscum album fermenté, collyres à l'hamamélis ou au plantain par exemple) comportent un contrôle optique avec évaluation de la limpidité dans le cas des solutions injectables. Une mesure du $\mathrm{pH}$ permet de s'assurer de la neutralité de la préparation. Une vérification de l'isotonie par détermination de l'abaissement cryoscopique, ainsi qu'un essai de stérilité (cf. contrôles microbiologiques) sont nécessaires. A cela s'ajoutent, dans le cas des préparations injectables, un essai d'apyrogénie, qui comporte une recherche de pyrogènes souvent chez le lapin et une recherche d'endotoxines bactériennes sur un lysat d'amoébocytes de limule (Limulus test), et dans le cas des collyres, une mesure de la viscosité. Si la préparation se présente sous la forme d'une suspension, il convient en outre de contrôler la taille des particules en suspension dans le but d'obtenir une suspension homogène.

Contrôle optique

Le contrôle optique d'une préparation injectable comprend d'une part le contrôle de son aspect, notamment de sa coloration, et d'autre part le contrôle de sa limpidité. Une 
modification de l'aspect initial témoigne de l'altération de la préparation. L'apparition d'une coloration anormale se détecte très facilement dans le cas des solutions incolores par un examen visuel du récipient posé sur un fond blanc. Dans le cas des solutions colorées, les changements de coloration sont décelés par comparaison avec un témoin et éventuellement, en utilisant une gamme étalon appropriée voire un électrophotomètre. Le contrôle de la limpidité ne s'applique par nécessité qu'aux solutions et non aux suspensions injectables. Il convient de préciser que la limpidité est toute relative puisqu'une solution optiquement vide n'existe pas, le nombre de particules décelées dépendant du système optique utilisé. La limite de taille des particules habituellement détectées par contrôle optique est de l'ordre de $100 \mu \mathrm{m}$ (Corriol et al., 2005 ; Tall et al., 2015 ; Blouet and Health, 2016 ; Ph.Eur., 2019a).

\section{Mesure du $\mathrm{pH}$}

Le pH joue un rôle important dans la fabrication des préparations injectables et des collyres puisqu'il conditionne la tolérance du médicament par l'organisme, sa stabilité et donc parfois son activité. Le pH du sang, de la lymphe et du liquide céphalo-rachidien est de l'ordre de 7,35 à 7,45 et celui du liquide lacrymal de 7,40 à 7,70. Il est cherché donc dans la formulation des préparations injectables et des collyres à ne pas trop s'éloigner de la neutralité. Cependant, pour des raisons d'instabilité, de précipitations de substances à $\mathrm{pH}$ neutre ou de variations d'activité selon le $\mathrm{pH}$, il est souvent nécessaire de s'éloigner de la neutralité. Le $\mathrm{pH}$ peut être ajusté en ajoutant une solution tampon. La mesure du $\mathrm{pH}$ s'effectue soit en utilisant des réactifs colorés, soit à l'aide d'un $\mathrm{pH}$-mètre. Il convient de préciser que le $\mathrm{pH}$ est susceptible de subir des modifications suite à des opérations telles que la filtration ou la stérilisation par la chaleur, ce qui nécessite de le contrôler avant et après ces opérations (Corriol et al., 2005; Tall et al., 2015; Blouet and Health, 2016; Ph.Eur., 2019a).

\section{Isotonie / Abaissement cryoscopique}

Afin d'éviter la survenue d'un phénomène d'hémolyse, il est nécessaire que les préparations injectables qui vont entrer en contact avec les liquides tissulaires aient la même pression osmotique donc la même osmolarité que ces derniers. De la même façon, les larmes présentent la même pression osmotique que le sang, il convient donc de préparer des collyres iso-osmolaires aux larmes, et ce d'autant plus que les yeux malades sont parfois plus sensibles aux variations de pression osmotique que les yeux sains, qui peuvent tolérer quant à eux, des concentrations en $\mathrm{NaCl}$ allant de $0,7 \%$ à $1,4 \%$. Il est possible d'évaluer directement l'isotonie par mesure de la pression osmotique. Cependant, cette mesure directe étant souvent difficile à réaliser pratiquement, il est préférable habituellement de faire recours à la détermination de l'abaissement cryoscopique de la solution : en effet, l'abaissement du point de congélation, tout comme la pression osmotique, varie proportionnellement au nombre de particules en solution. Il est de 0,52 ${ }^{\circ} \mathrm{C}$ dans le cas d'une solution isotonique à $0,9 \%$ de $\mathrm{NaCl}$ (Corriol et al., 2005 ; Tall et al., 2015; Blouet and Health, 2016 ; Ph.Eur., 2019a).

Apyrogénie

Les préparations injectables doivent être apyrogènes c'est-à-dire qu'elles ne doivent pas renfermer de substances appelées « pyrogènes » par SEIBERT en 1923, susceptibles de provoquer par injection une brusque élévation de température au-delà de 40 ${ }^{\circ} \mathrm{C}$ accompagnée de frissons intenses, d'une cyanose, d'une accélération du pouls, de céphalées et de douleurs lombaires. Les substances pyrogènes proviennent essentiellement de bactéries Gram négatives mais aussi de champignons inférieurs et de levures (Corriol et al., 2005 ; Tall et al., 2015; Blouet and Health, 2016 ; Ph.Eur., 2019a).

- Essai des pyrogènes (essai sur le lapin)

Cet essai n'est exigé par la Pharmacopée que dans le cas des préparations injectables de volume supérieur à $15 \mathrm{ml}$ ou si 
la mention « apyrogène » est apposée sur l'étiquette. Il consiste à suivre l'évolution de la température rectale de lapins auxquels la solution à tester a été injectée. Seuls sont retenus pour l'essai les lapins dont la sensibilité aux pyrogènes a été préalablement évaluée par injection d'une solution contenant des pyrogènes connus (Corriol et al., 2005; Tall et al., 2015; Blouet and Health, 2016; Ph.Eur., 2019a).

- Essai des endotoxines bactériennes (Limulus test)

Cet essai, également appelé essai LAL (pour « lysat d'amoebocytes de limule »), consiste à disposer sur une lame de verre un peu de réactif au lysat d'amoebocytes de limule Xiphosura polyphemus un arthropode marin, fouisseur, vivant au voisinage des côtes sur les fonds boueux (Antilles, océan Indien), appelé aussi improprement « crabe des Moluques » et un échantillon de la solution injectable à contrôler. La réaction est considérée comme positive si la viscosité augmente ou bien si le mélange coagule. Les résultats sont interprétés comparativement à un étalon d'endotoxine. Lorsque cet essai est pratiqué, l'essai des pyrogènes n'est pas exigé. Les études effectuées montrent en effet que l'essai sur le lapin et le Limulus test concordent presque toujours. La limite tolérée est exprimée en unités d'endotoxines par millilitre de solution. La Pharmacopée propose par ailleurs d'autres méthodes de recherche d'endotoxines bactériennes qui permettent en outre de quantifier ces substances : deux méthodes par gélification, deux méthodes par turbidimétrie et deux méthodes par colorimétrie (Corriol et al., 2005; Tall et al., 2015; Blouet and Health, 2016; Ph.Eur., 2019a). La technique de gélification permet la détection ou la quantification des endotoxines grâce à la propriété que possède le lysat de coaguler en présence d'endotoxines. $\mathrm{La}$ technique turbidimétrique consiste à mesurer l'accroissement de turbidité, par photométrie. Selon le principe expérimental mis en œuvre, la méthode utilisée est dite turbidimétrie en point final ou turbidimétrie cinétique. La technique colorimétrique consiste à mesurer la quantité de chromophore libéré par un peptide chromogène approprié lors de la réaction des endotoxines avec le lysat. Selon le principe expérimental mis en œuvre, la méthode utilisée est dite colorimétrie en point final ou colorimétrie cinétique. (Ph.Eur., 2008c)

Médicaments à base de plantes contenant exclusivement une ou plusieurs substances végétales (par exemple les préparations pour tisanes)

Cette catégorie concerne essentiellement les préparations pour tisanes, présentées en sachets-doses ou en vrac. Il convient alors de réaliser un essai de perte à la dessiccation, de vérifier la pureté en déterminant le taux d'éléments étrangers afin de déceler une éventuelle adultération ou falsification, de mesurer la taille des particules et, dans le cas des sachets-doses, d'effectuer un essai d'uniformité de dosage (masse et teneur).

\section{Suppositoires et ovules}

Dans le cas des suppositoires, les contrôles comportent un essai d'uniformité de masse, un contrôle de la dureté et de la résistance à la rupture, un essai de désagrégation et, dans le cas des suppositoires lipophiles (par exemple des suppositoires aux huiles essentielles), une détermination du temps de ramollissement. Dans le cas des ovules, il est réalisé un essai de désagrégation et un essai d'uniformité de dosage (masse et teneur) (Levacher, 2006).

Essais communs à de nombreuses formes galéniques

Essai d'uniformité de teneur

Cet essai vise à s'assurer que dans un échantillon de dix unités prélevées au hasard, les teneurs individuelles en principe actif, traceur d'activité ou traceur analytique se trouvent dans des limites raisonnables par rapport à la teneur moyenne de l'échantillon. Pour les comprimés, poudres à usage parentéral et suspensions injectables, l'ensemble des dix valeurs doit se situer dans un intervalle allant de $85 \%$ à $115 \%$ de la moyenne ; il est toutefois 
toléré qu'une valeur sorte de cet intervalle mais se situe entre $75 \%$ et $125 \%$ de la moyenne, à condition qu'en refaisant l'essai sur vingt autres unités, la totalité des trente unités testées se situent entre $75 \%$ et $125 \%$ de la moyenne. Pour les capsules, poudres à usage non parentéral, granulés, suppositoires et ovules, l'ensemble des dix valeurs doit se situer dans un intervalle allant de $85 \%$ à $115 \%$ de la moyenne ; il est toutefois toléré qu'une valeur sorte de cet intervalle mais se situe entre $75 \%$ et $125 \%$ de la moyenne; si deux ou trois valeurs sont dans ce cas, il est alors nécessaire de refaire l'essai sur vingt autres unités et seules trois valeurs sur le total des trente peuvent sortir de l'intervalle [85\% - 115\%] sans sortir de l'intervalle $[75 \%-125 \%]$ (Brossard et al., 2016).

Essai d'uniformité de masse (2.9.5) : Ph. Eur

Cet essai vise à s'assurer que sur vingt unités prélevées au hasard, les masses individuelles se trouvent dans des limites raisonnables par rapport à la masse moyenne. Il convient de noter que la tolérance est plus grande pour les petites unités du fait de l'erreur relative supérieure. Deux valeurs sur les vingt mesurées peuvent être supérieures à l'écartlimite sans toutefois excéder le double de cet écart-limite (Brossard et al., 2016).

Tableau 1 : Voies d'administration et formes des médicaments.

\begin{tabular}{ll}
\hline Voies d'administration & Formes principales \\
\hline Orale & Comprimés, gélules, solutions ou suspensions aqueuses \\
Parentérale & Solutions aqueuses \\
Rectale & Suppositoires \\
Vaginale & Comprimés, solutions aqueuses \\
Ophtalmique & Solutions aqueuses, pommades. \\
ORL & Solutions aqueuses pulvérisées ou non \\
Percutanée & Pommades, crème et solutions. \\
\hline
\end{tabular}

Tableau 2 : Méthodes d'études phytochimiques.

\begin{tabular}{|c|c|c|}
\hline Groupes phytochimiques & Test en tube & Test par CCM \\
\hline Huiles essentielles et matières grasses & Détection de l'odeur & \\
\hline Stérols et triterpènes & Liebermann-Burchard & $\begin{array}{l}\text { Vanilline sulfurique et } \\
\text { Libermann Burchard }\end{array}$ \\
\hline Caroténoïdes & Carr Price & \\
\hline Coumarines & Feigl sous une lampe UV & $\mathrm{KOH}$ méthanolique à $5 \%$ \\
\hline Alcaloïdes & Dragendorff & Dragendorff \\
\hline Flavonoïdes ou des aglycones flavoniques & cyanidine & $\mathrm{Neu}$ \\
\hline Tanins & $\mathrm{FeCl}_{3} 2 \%$ & $\mathrm{FeCl}_{3}$ \\
\hline Saponosides & Présence de mousse & Anisaldehyde sulfurique \\
\hline
\end{tabular}


Tableau 3: Liste des pesticides.

\begin{tabular}{|c|c|}
\hline Substance & Tolérance $(\mathrm{mg} / \mathrm{kg})$ \\
\hline Alachlor & 0,02 \\
\hline Aldrine et Dieldrine (somme de) & 0,05 \\
\hline Azinfos-méthyle & 1,0 \\
\hline Bromopropylate & 3,0 \\
\hline Chlordane (somme des isomères cis-, trans-et oxychlordane) & 0,05 \\
\hline Chlorfenvinphos & 0,5 \\
\hline Chlorpyrifos & 0,2 \\
\hline Chlorpyrifos-méthyle & 0,1 \\
\hline Cyperméthrine (et isomères) & 1,0 \\
\hline DDT (somme de p,p'-DDT, o,p'-DDT, p,p'-DDE et p,p'-TDE & 1,0 \\
\hline Deltaméthrine & 0,5 \\
\hline Diazinon & 0,5 \\
\hline Dichlorvos & 1,0 \\
\hline Dithiocarbamates (enCS2) & 2,0 \\
\hline Endosulfan (somme des isomères et du sulfate d'endosulfan) & 3,0 \\
\hline Endrine & 0,05 \\
\hline Ethion & 2,0 \\
\hline Fenitrothion & 0,5 \\
\hline Fenvalerate & 1,5 \\
\hline Fonofos & 0,05 \\
\hline Heptachlor (somme d'heptachlor et d'heptachlorépoxyde) & 0,05 \\
\hline Hexachlorobenzène & 0,1 \\
\hline Hexachlorocyclohexane-Isomères (autres que $\gamma$ ) & 0,3 \\
\hline Lindane ( $\gamma$-Hexachlorocyclohexane) & 0,6 \\
\hline Malathion & 1,0 \\
\hline Méthidathion & 0,2 \\
\hline Parathion & 0,5 \\
\hline Parathion-méthyle & 0,2 \\
\hline Perméthrine & 1,0 \\
\hline Phosalone & 0,1 \\
\hline Pipéronylbutoxyde & 3,0 \\
\hline Pirimiphos-méthyle & 4,0 \\
\hline Pyréthrines (somme des) & 3,0 \\
\hline $\begin{array}{l}\text { Quintozène (somme de quintozène, pentachloroaniline et } \\
\text { pentachlorophénylsulfure de méthyle) }\end{array}$ & 1,0 \\
\hline
\end{tabular}


Tableau 4 : Limites des paramètres microbiens.

\begin{tabular}{ll}
\hline Analyse de l'impureté microbienne & Limites de tolérances \\
\hline Recherche de germes totaux & \\
Numération sur plaque des microorganismes aérobies & $10^{4} \mathrm{UFC} / \mathrm{g} \mathrm{ou} \mathrm{mL}$ \\
Numération des levures et moisissures (champignons) & $10^{2} \mathrm{UFC} / \mathrm{g} \mathrm{ou} \mathrm{mL}$ \\
Recherche de germes spécifiques & \\
Enterobacteriaceae et bactéries Gram-négatif tolérantes à la bile & $10^{2} \mathrm{UFC} / \mathrm{g}$ ou mL \\
Salmonella spp & Absent $(10 \mathrm{~g} \mathrm{ou} 10 \mathrm{~mL})$ \\
Escherichia coli & Absent $(1 \mathrm{~g} \mathrm{ou} 1 \mathrm{~mL})$ \\
Staphylococcus aureus & Absent $(1 \mathrm{~g} \mathrm{ou} 1 \mathrm{~mL})$ \\
Pseudomonas aeruginosa & Absent $(1 \mathrm{~g}$ ou $1 \mathrm{~mL})$ \\
\hline
\end{tabular}

Tableau 5 : Solvants de différentes classes dans les produits à usage pharmaceutique.

\begin{tabular}{|c|c|c|}
\hline Classe 1 & Classe 2 & Classe 3 \\
\hline Benzène & Acétonitrile & Acide acétique \\
\hline Tétrachlorure de carbone & Chlorobenzène & Heptane \\
\hline 1,2-Dichloroéthane & Chloroforme & Acétone \\
\hline 1,1-Dichloroéthène & Cyclohexane & Acétate d'isobutyle \\
\hline \multirow[t]{23}{*}{ 1,1,1-Trichloroéthane } & 1,2-Dichloroéthène & Anisole \\
\hline & Dichlorométhane & Acétate d'isopropyle \\
\hline & 1,2-Diméthoxyéthane & 1-Butanol \\
\hline & N,N-Diméthylacétamide & Acétate de méthyle \\
\hline & N,N-Diméthylformamide & 2-Butanol \\
\hline & 1,4-Dioxane & 3-Méthyl-1-butanol \\
\hline & 2-Ethoxyéthanol & Acétate de butyle \\
\hline & Ethylèneglycol & Méthyléthylcétone \\
\hline & Formamide & tert-Butylméthyléther \\
\hline & Hexane & Méthylisobutylcétone \\
\hline & Méthanol & Cumène \\
\hline & 2-Méthoxyéthanol & 2-Méthyl-1-propanol \\
\hline & Méthylbutylcétone & Diméthylsulfoxyde \\
\hline & Méthylcyclohexane & Pentane \\
\hline & N-Méthylpyrrolidone & Ethanol \\
\hline & Nitrométhane & 1-Pentanol \\
\hline & Pyridine & Acétate d'éthyle \\
\hline & Sulfolane & 1-Propanol \\
\hline & Tétrahydrofurane & Ether éthylique \\
\hline & Tétraline & 2-Propanol \\
\hline & Toluène & Formate d'éthyle \\
\hline & 1,1,2-Trichloroéthène & Acétate de propyle \\
\hline & Xylène & Acide formique \\
\hline
\end{tabular}

\section{CONCLUSION}

La médecine traditionnelle africaine et les praticiens de la santé traditionnelle pourraient apporter une contribution significative à la réalisation de la couverture sanitaire universelle. L'utilisation de médicaments à base de plantes issues de cette médecine pour des soins primaires occupe une place importante dans le système de santé des pays africains surtout subsahariens. Des nombreuses études confirment que le recourt aux produits issus des plantes médicinales 
comme alternatives aux soins de santé est en pleine croissances ces dernières années. Ce constat répond à des exigences tant économiques que socio-culturelles. Cette augmentation a aussi ses corollaires et conduit à diverses formes d'abus liés à des facteurs extrinsèques au produit telles les falsifications, le mauvais traitement et conservation des produits, qui souvent, mènent à la déception des consommateurs et des fabricants. Ce qui explique en partie la méfiance des acteurs de la santé et la très faible prescription des médicaments à base de plantes. Les défis deviennent énormes et obligent les Etats et l'OMS à sécuriser son utilisation. Cette étude a permis de résumer les différents outils nécessaires à l'établissement des paramètres de contrôle qualité utiles à tous les acteurs de la chaîne de production de médicaments à base de plantes. L'utilisation de ces paramètres permettra de garantir une standardisation et une validation des procédés utilisés au cours de la production. Ces étapes permettent d'aboutir à des produits de qualité constante et conforme aux exigences réglementaires.

\section{CONFLIT D'INTERETS}

Les auteurs déclarent qu'il n'y a aucun conflit d'intérêts.

\section{CONTRIBUTIONS DES AUTEURS}

SO, JY, TKT et MN sont intervenus dans toutes les phases de l'étude. Il s'agit de la conception de l'étude, la revue de la littérature, l'analyse des données de la revue et la rédaction du présent manuscrit. BCS, HZD, JBGY et $\mathrm{AD}$ sont intervenus dans la conception de l'étude et la correction du manuscrit. LB, FBK, SO et RS sont intervenus dans la conception de l'étude et dans la correction du manuscrit.

\section{REMERCIEMENTS}

Les auteurs remercient toute les Professeurs Sylvin Ouédraogo, Rasmané Semdé ainsi que leurs équipes de l'Institut de recherche en science de la santé (IRSS) et du Centre de Formation, de Recherche et d'Expertises en Sciences du Médicament (CEA-CFOREM) de l'Université Joseph KIZERBO au Burkina Faso qui ont mis à notre disposition une base des données et auprès de qui une grande partie de la documentation (sources primaires) a été obtenue.

\section{REFERENCES}

Agaie B, Onyeyili P, Muhammad B, Landan M. 2007. Some toxic effects of aqueous leaf extract of Anogeissus leiocarpus in rats. Journal of Pharmacology and Toxicology, 2: 396-401.

AOAC. 2005. Official Methods of Analysis of AOAC International. AOAC International: Gaithersburg (MD).

Bekro Y-A, Mamyrbekova J, Boua BB, Bi FT, Ehile EE. 2007. Etude ethnobotanique et screening phytochimique de Caesalpinia benthamiana (Baill.) Herend. et Zarucchi (Caesalpiniaceae). Sciences \& Nature, 4 : 217-225.

DOI:

10.4314/scinat.v4i2.42146

Bellenot D, Toure H, Garinot O, Le Roux E, L'Huillier-Chaigneau A. 2012. Problématiques liées au développement d'une procédure analytique de dosage de constituants dans les médicaments à base de plante (s) I. Données de la littérature et commentaires. STP Pharma Pratiques, 22: 3-15.

Blouet E, Health GBUP. 2016. Parenteral Preparations, Challenges in Formulations. Roquette Pharma: Lestrem, France.

Bodeker G, Ong C-K. 2005. WHO global atlas of traditional, complementary and alternative medicine. World Health Organization, Genève, Suisse.

Brossard D, Charrueau C, Chaumeil J-C, Crauste-Manciet S, Le Hir A. 2016. Pharmacie Galenique: Bonnes Pratiques de Fabrication des Medicaments. Elsevier Masson: Paris, France.

Calixto J. 2000. Efficacy, safety, quality control, marketing and regulatory guidelines for herbal medicines (phytotherapeutic agents). Brazilian Journal of Medical and Biological Research, 33: 179-189. DOI: 10.1590/s0100-879x2000000200004

Chaaib Kouri F. 2004. Investigation phytochimique d'une brosse à dents africaine "Zanthoxylum zanthoxyloides" (Lam.) Zepernick et Timler (Syn. "Fagara zanthoxiloides" L.) (Rutaceae). Thèse de Doctorat, Faculté des Géosciences et de l'Environnement, Université de Lausanne, $200 \mathrm{p}$. 
Chabrier JY. 2010. Plantes médicinales et formes d'utilisation en Phytothérapie. Thèse de Pharmacie, Sciences du Vivant [q-bio] / Sciences Pharmaceutiques, Université Henri Poincare, Nancy 1, 184 p.

Chavass D, Kolwicz C, Smith B. 2001. Médicaments Essentiels : le Point No. 030. Bibliothèque des Systèmes de Santé de l'OMS, 27: 1-27.

Chegaing SPF, Mefokou DY, Tangue BT, Sokoudjou JB, Menoudji ST, Kamsu TG, Gatsing D. 2020. Contribution to the ethnobotanical inventory of medicinal plants used for the treatment of typhoid fever in Adamaoua region, Cameroon. International Journal of Biological and Chemical Sciences, 14(9): 3078-3096. DOI: $10.4314 /$ ijbcs.v14i9.9

Corriol O, Crauste-Manciet S, Arnaud P, Brion F, Brossard D, Causse R, Chaumeil J-C, Collomp R, Cynober L, Darbord J-C, de Launet Q, Deprez M, Gard C, Lagarde A, Mazère J, Meunier P, Navas D, Neuville $\mathrm{S}$, Préaux N, Provot S, Rambourg $\mathrm{P}$, Saubion J-L, Yona YT. 2005. Recommandations pour la préparation des mélanges de nutrition parentérale. Nutrition Clinique et Métabolisme, 19(1): 30-55.

DOI: https://doi.org/10.1016/j.nupar.2005.01.0 03

Council UEAS. 2009. Potential of traditional medicine should be fostered, Economic and Social Council President tells panel on attaining Millennium Development Goals in public health. ECOSOC/6385, Department of Public Information, News and Media Division, New York.

Da O, Coulibaly MT, Ouédraogo J, Yaro B, Yerbanga R, Kini F, Koama B, Dakuyo Z, Nikiema J, Ouédraogo G. 2015. Phytochemical screening of Saye, a traditional herbal remedy for malaria. International Journal of Biological and Chemical Sciences, 9(6): 2940-2946. DOI: 10.4314/ijbcs.v9i6.33

Dongock DN, Bonyo AL, Mapongmestem PM, Bayegone E. 2018. Etude ethnobotanique et phytochimique des plantes médicinales utilisées dans le traitement des maladies cardiovasculaires à Moundou (Tchad). International Journal of Biological and
Chemical Sciences, 12(1): 203-216. DOI: 10.4314/ijbcs.v12i1.16

DPSNSO. 2015. Guide de référence sur la qualité des produits de santé naturels. DPSNSO, Canada.

EMA. 2002. Points to Consider on Good Agricultural and Collection Practice for Starting Materials of Herbal Origin. EMA, Amsterdam, Pays-Bas.

EMA. 2005. Guideline on Quality of Herbal Medicinal Products/Traditional Herbal Medicinal Products. EMA, Amsterdam, Pays-Bas.

Evans WC. 2002. Trease and Evans' Pharmacognosy. Saunders Company Limited: London.

FAO. 2018. Commission du codex alimentarius : Programme mixte FAO/OMS sur les normes alimentaires comité du codex sur les contaminants dans les aliments. FAO, Utrecht, PaysBas.

Fatiha S. 2019. Pharmacognosie. Mémoire de Master 1 en Génie Pharmaceutique, Universite des Sciences et de la Technologie d'Oran «Mohamed Boudiaf», Algerie, $62 \mathrm{p}$.

Franz G, Wang M. 2015. The role of the European Pharmacopoeia ( $\mathrm{Ph}$ Eur) in quality control of traditional Chinese herbal medicine in European member states. World Journal of Traditional Chinese Medicine, 1: 5-15. DOI: 10.15806/j.issn.2311-8571.2014.0021

Hubert P, Nguyen-Huu J-J, Boulanger B, Chapuzet E, Chiap P, Cohen N, Compagnon PA, Dewé W, Feinberg M, Lallier M. 2003. Validation des procédures analytiques quantitatives, Harmonisation des démarches. STP Pharma Pratiques, 13: 101-138.

ICH. 2003. Ligne directrice : Essais de stabilité de nouveaux produits et substances édicamenteux - ICH thème Q1A(R2). $\mathrm{ICH}$, Direction Générale des Produits de Santé et des Aliments.

Isérin P, Masson M, Kedellini J. 2001. Encyclopédie des Plantes Médicinales, Identifications, Préparations. Edition Larousse/VUEF 335: Paris.

Kabré E, Bandé M, Karfo R, Bazié R, Soulama D, Nikiéma F, Sakandé J. 2020. Quantitative determination of chemical and biochemical parameters during 
«soumbala» manufacturing process in two communities of Burkina Faso. International Journal of Biological and Chemical Sciences, 14(9): 3001-3017. DOI: 10.4314/ijbcs.v14i9.4

Kasilo OMJ, Wambebe C, Nikiema JB, Nabyonga-Orem J. 2019. Towards universal health coverage: advancing the development and use of traditional medicines in Africa. BMJ Global Health, 4: e001517. DOI:10.1136/bmjgh-2019001517

Khandelwal KR. 2008. Practical Pharmacognosy. Pragati Books Pvt. Ltd: Pune, Inde.

Kokate CK, Purohit AP, okhale SB. 2004. Text Book of Pharmacognosy (27 $7^{\text {th }}$ edn). Pragati Books Pvt. Ltd: Pune, India.

Le Hir A. 2009. Vie d'un Médicament, de la Conception aux Bonnes Pratiques de Fabrication: Abrégés de Pharmacie Galénique Bonnes Pratiques de Fabrication des Médicaments. Edition Masson: Paris, France.

Lehmann H. 2013. Le médicament à base de plantes en Europe: statut, enregistrement, contrôles. Thèse de Doctorat en Sciences Pharmaceutiques, Université de Strasbourg, Strasbourg, 342 p.

Levacher E. 2006. Méthodes générales d'analyse des formes solides. In Phamaco-Technie Industrielle. IMT Editions: Tours, France ; 423-431.

Limonier A-S. 2018. La phytothérapie de demain: les plantes médicinales au cœur de la pharmacie. Thèse de Doctorat en Sciences Pharmaceutiques, Faculté de pharmacie, Aix-Marseille Université, Marseille, 99 p.

Mamyrbékova-Békro JA, Konan KM, Békro Y-A, Gobin M, Bi D, Bi T, Mambo V, Boua Boua B. 2008. Phytocompounds of the extracts of four medicinal plants of Côte D'ivoire and assessment of their potential antioxidant by thin layer chromatography. Eur. J. Scient. Res., 24: 219-228.

Miningou A, Golane V, Traore AS, Kambire H. 2020. Determination of the optimal dose and date of application of mineral manure on sesame (sesamum indicum L.) in Burkina Faso. International Journal of
Biological and Chemical Sciences, 14(9): 2992-3000. DOI: 10.4314/ijbcs.v14i9.3

Mokosso JdDM, van Diggelen R, Mwanga JC, Malaisse F, Robbrecht E. 2012. Etude ethnoptéridologique, évaluation des risques d'extinction et stratégies de conservation aux alentours du Parc National de Kahuzi Biega (RD Congo). Geo-Eco-Trop., 36: 137-158.

Mukherjee PK. 2002. Quality Control of Herbal Drugs: An Approach to Evaluation of Botanicals. Business Horizons: Michigan.

OMS. 2013. Stratégie de l'OMS pour la médecine traditionnelle pour 2014-2023. Organisation mondiale de la Santé, Suisse.

Ouédraogo B, Yoda J, Kini BF, Koala M, Yaro A, Bonzi-Coulibaly Y. 2019. Phytochemical screening and in vitro antioxidant study of six plants used for the treatment of hypertension in traditional medicine. World Journal of Pharmacy and Pharmaceutical Sciences, 8(4): $\quad$ 1665-1678. DOI: 10.20959/wjpps20194-13559

Patel P, Patel N, Goyal R. 2006. Quality control of herbal products. The Indian Pharmacists, 5: 26-30.

Ph.Eur. 2008a. European Directorate for the Quality of Medicines and HealthCare (EDQM) of the Council of Europe. European Pharmacopoeia. Ph.Eur.: Strasbourg, France.

Ph.Eur. 2008b. Pharmacopée Européenne ( $8^{\text {th }}$ edn). Ph.Eur.-Conseil d'Europe: Stratbourg.

Ph.Eur. 2008c. Pharmacopée Européenne (9 $9^{\text {th }}$ edn). Ph.Eur.-Conseil d'Europe: Stratbourg.

Ph.Eur. 2011. European Pharmacopoeia : Technical Guide for the Elaboration of Monographs ( $6^{\text {th }}$ edn). Ph.Eur.-European Directorate for the Quality of Medicines \& HealthCare: Stratbourg.

Ph.Eur. 2016. Pharmacopée Européenne (9ème édn). Ph.Eur.-European Directorate for the Quality of Medicines $\&$ HealthCare: Stratbourg.

Ph.Eur. 2019a. Pharmacopée Européenne (10ème édn). Ph.Eur.-European Directorate for the Quality of Medicines $\&$ HealthCare: Stratbourg. 
Ph.Eur. 2019b. Pharmacopée Européenne (10ème édn). Ph.Eur.-European Directorate for the Quality of Medicines $\&$ HealthCare: Stratbourg.

Ph.Fr. 2012. Monographie. Ph.Fr.: France.

Pinguet I. 2015. Validation analytique: application de la procédure SFSTP 20032006 au domaine de la phytothérapie. Mémoire de Master, Sciences Pharmaceutiques, UB - Université de Bordeaux, P14.

Raynaud M. 2011. Validation du procédé de fabrication dans l'industrie pharmaceutique appliquée aux formes solides orales. Thèse de Doctorat, Université de Limoges. Faculté de médecine et de pharmacie, Limoges.

Richard D, Senon J-L, Valleur M. 2004. Dictionnaire des Drogues et des Dépendances. Larousse: Paris, France.

Siddiqui S, Verma A, Rather AA, Jabeen F, Meghvansi MK. 2009. Preliminary phytochemicals analysis of some important medicinal and aromatic plants. Advances in Biological Research, 3: 188195.

Société Française. 2007. Ethnopharmacologia. Société Française d'Ethnopharmacologie.

Sofowora A. 2010. Plantes Médicinales et Médecine Traditionnelle d'Afrique: KARTHALA Editions: Paris, France.

Tall ML, Salmon D, Diouf E, Drai J, Filali S, Lépilliez V, Pioche M, Laleye D, Dhelens C, Ponchon T, Pivot C, Pirot F. 2015. Validation du procédé aseptique et étude de stabilité d'une préparation injectable de fructose (5\%)-glycérol (10\%) dans le cadre d'un programme hospitalier de recherche clinique portant sur le traitement curatif endoscopique des lésions néoplasiques épithéliales précoces $\mathrm{du}$ tube digestif. Annales Pharmaceutiques Francaises, 73: 139149.

Tarpaga WV, Bourgou L, Guira M, Rouamba A. 2020. Agro morphological characterization of cashew trees (Anacardium occidental L.), in improvement for the high yield and high quality of raw nuts in Burkina Faso. International Journal of Biological and Chemical Sciences, 14(9): 3188-3199. DOI: 10.4314/ijbcs.v14i9.17
USP. 2007. Pharmacopées Americaine. USP, Suisse.

Wehrlé P. 2007. Contrôle biopharmaceutique des formes orales solides. Pharmacie galénique, formulation et technologie pharmaceutique Maloine, Maloine.

WHO. 2000a. General guidelines for methodologies on research and evaluation of traditional medicine. World Health Organization, Genève, Suisse.

WHO. 2000b. Principes méthodologiques généraux pour la recherche et l'évaluation relatives à la médecine traditionnelle. Organisation mondiale de la Santé, Genève, Suisse.

WHO. 2002a. General guidelines for methodologies on research and evaluation of traditional medicine. World Health Organization, Genève, Suisse..

WHO. 2002b. Stratégie de l'OMS pour la médecine traditionnelle pour 2002-2005. Organisation mondiale de la Santé, Genève, Suisse.

WHO. 2003a. Directives OMS sur les bonnes pratiques agricoles et les bonnes pratiques de récolte (BPAR) relatives aux plantes médicinales. Organisation mondiale de la Santé, Genève, Suisse.

WHO. 2003b. WHO guidelines on good agricultural and collection practices [GACP] for medicinal plants. World Health Organization, Genève, Suisse.

WHO. 2004. Guidelines on good agricultural and collection practices (GACP) for medicinal plants. World Health Organization, Genève, Suisse.

WHO. 2007. WHO guidelines for assessing quality of herbal medicines with reference to contaminants and residues. World Health Organization, Genève, Suisse.

WHO. 2019. The International Pharmacopoeia. World Health Organization, Genève, Suisse.

Wichtl M, Anton R, Bernard M, Czygan F-C. 2003. Plantes Thérapeutiques: Tradition, Pratique Officinale, Science et Thérapeutique. Ed. médicales internationales: Ouagadougou Burkina Faso.

Yadav N, Dixit V. 2008. Recent approaches in herbal drug standardization. Int. J. Integr. Biol., 2: 195-203. 